\title{
Safety of human olfactory mucosal biopsy for the purpose of olfactory ensheathing cell harvest and nerve repair: a prospective controlled study in patients undergoing endoscopic sinus surgery*
}

\author{
Peter J. Andrews ${ }^{1,4}$, Anne-Lise Poirrier ${ }^{2}$, Valerie J. Lund ${ }^{1,4}$, David Choi ${ }^{3}$ \\ ' Department of Rhinology and Facial Plastic Reconstructive Surgery, The Royal National Throat, Nose and Ear Hospital, \\ London, United Kingdom \\ 2 ENT Department, University Hospital of Liege, Sart-Tilman B35, Liege, Belgium \\ ${ }^{3}$ Department of Neurosurgery, The National Hospital for Neurology and Neurosurgery, Queen Square, London, United Kingdom \\ ${ }^{4}$ EAR Institute, UCL, London, United Kingdom
}

Rhinology 54: 183-191, 2016

DOI:10.4193/Rhino15.365

*Received for publication:

December 17, 2015

Accepted: January 21, 2016

\begin{abstract}
Background: Nasal olfactory mucosa is an accessible source of olfactory ensheathing cells for spinal cord regeneration. However, safety of the biopsy technique and the effects on sense of smell and nasal function have not been robustly assessed in the form of a prospective controlled study.
\end{abstract}

Methodology: National Health Service ethical approval was granted for this study of 131 patients. The primary outcome measure was olfactory function and the secondary outcomes included postoperative complication rates as well as the SNOT 22, NOSE scale scores and surgeon reported (Lund-Kennedy score) nasal function outcomes.

Results: 65 patients underwent functional endoscopic sinus surgery (FESS) and superior turbinate biopsy, and 66 patients underwent FESS only as the control group. There was no significant difference in complication rates between the two groups. All Olfactory function outcomes were unaffected following olfactory biopsy. We demonstrated that the patient's quality of life and nasal patency as well as surgeon reported outcome measurements remain unaffected following olfactory harvesting.

Conclusions: We have uniquely provided level 2a evidence for the safety of endoscopic biopsy of olfactory mucosa, which does not affect nasal function or the sense of smell compared to standard FESS without biopsy.

Key words: smell, nasal surgery, rhinitis, olfactory mucosa, glia, quality of life

\section{Introduction}

The nasal olfactory mucosa is an accessible source of Olfactory Ensheathing Cells (OECs) which can be harvested for spinal cord and brachial plexus repair. Current clinical studies utilizing OECs in central nervous system (CNS) regeneration have shown promising results ${ }^{(1-3)}$. In order to move into clinical trials of OECs for nerve repair there is a need to perform olfactory mucosa harvest procedures ${ }^{(4)}$. Current evidence suggests that human olfactory mucosal biopsies do not have a detrimental effect on the patient's sense of smell ${ }^{(5,6)}$, however up to now a robust prospective controlled study assessing olfaction, quality of life and patient and surgeon reported outcome measurements has not been preformed.

Repair of the human central nervous system (CNS) following trauma remains a surgical challenge. The human olfactory 
system may provide an answer by utilizing the regenerative ability of the olfactory ensheathing cell (OEC), which stimulates the natural neurogenesis of the human olfactory nerve fibres. The concept of using OECs for CNS repair is predicated on their intrinsic ability to facilitate neurogenesis. OECs could be harvested from the olfactory bulb but would be associated with a risk of stroke, seizures or death ${ }^{(7-9)}$. A safer technique is to harvest nasal olfactory mucosa endonasally which equally contains OECs, but its safety has not been fully evaluated (4). Surgical techniques for nasal olfactory harvesting have been reported, however patient numbers studied are small and lack extensive safety analysis ${ }^{(10,11)}$.

Olfactory ensheathing cells (OECs) are supportive glial cells for the olfactory receptor neurons (ORNs) and are found both in the olfactory bulb of the CNS and the olfactory mucosa of the PNS ${ }^{(12,13)}$. They were described by Doucette in 1984 as a distinct glial cell entity enabling regeneration of the olfactory system ${ }^{(14,15)}$. They support, nurture and facilitate regeneration of the olfactory nerves and have the unique ability to transgress both PNS and CNS environments ${ }^{(16)}$. They also provide important neuro-protective properties and stimulate regeneration through neurotrophic signaling and myelination of new axons, providing a continuous channel for the regeneration of new olfactory axons ${ }^{(8)}$. OECs have the unique ability to interact and migrate within the astrocytic rich environment of the CNS and human olfactory bulb ${ }^{(17)}$. OECs have been shown to also re-myelinate de-myelinated CNS axons ${ }^{(13)}$.

The aim of this study is to assess patient safety, in terms of post operative complication rates and nasal function morbidity after excision of the middle section of the superior turbinate, using our previously described technique compared to a control group, from a cohort of patients undergoing endoscopic sinus surgery (ESS) for CRS or other diseases ${ }^{(4)}$. The potential complications assessed included haemorrhage, infection, and cerebrospinal fluid (CSF) leakage. Nasal function morbidity was assessed using both patient and surgeon reported nasal function outcomes, particularly focusing on the sense of smell. The primary endpoint was olfaction measured by the University of Pennsylvania Smell Identification Test (UPSIT). As well as patient reported outcomes, we assessed surgeon reported outcomes, of the appearance of nasal mucosa during the healing process. This work has been performed in connection with our research on OEC culture techniques with a view to performing clinical trials in the future ${ }^{(4,18)}$.

\section{Materials and methods}

Ethical approval and consent

Ethical approval was granted by the NHS Research Ethics Committee to obtain biopsies from patients scheduled for routine endoscopic sinus surgery rather than healthy volunteers. Therefore our biopsy group consisted of patients having endoscopic sinus surgery (ESS) compared to a control group undergoing ESS only. Informed consent was obtained for participation in this study in addition to the consent for the Functional Endoscopic sinus surgery (FESS) operation. The aims of the study, risk factors of the procedure and the absence of direct benefit to the patients themselves were discussed prior to obtaining consent. Patients who refused a biopsy were subsequently entered into the control arm of the study.

\section{Inclusion and exclusion criteria}

The 131 patients were recruited from the Royal National Throat Nose and Ear Hospital over a 2 year period. Those requiring FESS for the treatment of a broad range of sinus diseases including benign neoplasia were invited to participate in this prospective study. The exclusion criteria included patients under the age of 16 , pregnancy and the inability to comprehend the assessment questionnaires. Those requiring FESS for the treatment of chronic rhinosinusitis were listed in accordance to the European Position Paper on Rhinosinusitis and Nasal Polyps (EPOS) guidelines ${ }^{(19)}$ and non-infective pathologies including the excision of benign neoplasia were also invited to participate. All our CRS patients were refractory to optimum nasal medication and therefore listed for FESS to improve the ventilation of the sinuses and restore mucociliary clearance. The patients in this study presented with a combination of symptoms including nasal obstruction, reduction in sense of smell, rhinorrhea or facial pain.

\section{UPSIT measured outcome}

Olfaction was measured using both the University of Pennsylvania Smell Identification Test (UPSIT) which is a 40 item psychophysical scratch and sniff test as well as assessing the patient's perceived sense of smell using the visual analogue scale. According the UPSIT scores, hyposmic patients are classified into mild, moderate and severe; male mild 30-33, moderate 26-29, severe 19-25, females; mild 31-34, mod 26-30, severe 19-25 (20).

\section{Outcome measurements}

Our outcome measures included complication rates, patient and surgeon recorded outcome measurements. Patient safety was assessed through the evaluation of both intra and post operative complication rates including bleeding, infection and CSF leakage rates. The immediate adverse safety outcomes evaluated during the biopsy were hemorrhage and cerebrospinal fluid leakage. This was assessed at the time of surgery and immediately post-operatively. Secondary hemorrhage and infection were assessed at the 3 week follow up appointment.

The secondary endpoint measurements included; the LundKennedy Staging System which is a surgeon reported endosco- 
pic nasal mucosa outcome measure (21), and an assortment of patient reported outcome measures (PROM) which included the sinonasal outcome test (SNOT-22) which is a disease specific quality of life measure ${ }^{(22)}$, and the symptom specific PROMs which include the global nasal function visual analogue scale (VAS) and the Nasal Obstruction and Symptom Effectiveness (NOSE) Score. Patients were evaluated pre operatively, intra operatively and post operatively both in the immediate post operative recovery and subsequent follow-up at 6 months. We compared our findings with a control group who underwent similar ESS surgery but without an olfactory mucosal biopsy. The patient's medical history was documented including age, sex, presenting complaint, past medical and surgical history, drug history, allergies and smoking status. In addition, recent use of topical and/or oral steroids was documented.

\section{Lund Mackay grading system}

All patients undertook a CT scan pre operatively. Disease severity was objectively assessed on CT scans and subsequently graded according to severity based on sinus opacification using the Lund Mackay scoring system ${ }^{(23)}$. This staging system bilaterally scores the five sinuses from zero to two, depending on severity of opacification. " 0 " is assigned to a sinus without opacification and " 2 " is assigned to complete opacification, and "1" for partial opacification. The ostiomeatal complex (OMC) is also scored but either one or two (presence or absence of opacification). The grading score ranges from zero to 24 .

\section{VAS score}

The Visual Analogue Scale (VAS) for global nasal symptoms is an EPOS recommended measurement for determining disease severity ${ }^{(19)}$. It allows patients to subjectively rate their symptoms on a $10 \mathrm{~cm}$ linear scale, where 0 corresponds to no symptoms and 10 is the most severe. A score of 5 is generally considered significant. VAS scores were measured for nasal blockage and sense of smell.

\section{SNOT-22}

The validated SNOT $22 \mathrm{QOL}$ score is one of the most widely used disease specific quality of life questionnaires which was originally designed for the assessment of sinonasal disease ${ }^{(22)}$, and has also been validated for a broader range of nasal disorders including patients undergoing septal and septorhinoplasty surgery ${ }^{(24)}$.

\section{Nose questionnaire}

The NOSE questionnaire provides a validated symptom specific questionnaire, which specifically assesses the symptom of nasal obstruction and its consequences ${ }^{(25)}$. This brief questionnaire consists of five questions, which are used to rate the burden of nasal obstruction during the past month, with each question scored from 0 (not a problem) to 4 (severe problem); nasal congestion or stuffiness, nasal blockage or obstruction, trouble breathing through my nose, trouble sleeping and unable to get enough air through my nose during exercise or exertion. A maximum score of 20 is then multiplied by 5 to come to a potential 100.

\section{Lund-Kennedy score}

The Lund-Kennedy endoscopic scoring system is subjectively scored by the surgeon and quantifies the pathologic state of the paranasal sinuses and in doing so quantifies the severity of polyps, discharge, edema, scarring or adhesions and crusting and the score ranges from 0 to $20^{(21)}$. Polyps are graded as absent (0), present in the middle meatus (1), or present beyond the middle meatus (2). Discharge is graded as not present (0), thin (1), or thick and purulent (2). Edema, scarring, and crusting are each graded as absent (0), mild (1), or severe (2). Each questionnaire requires the patient to measure their symptom severity and its impact on their quality of life at that specific moment.

\section{Biopsy technique}

As previously described the middle section of the superior turbinate was excised through two horizontal incisions fashioned with curved endoscopic forceps and completed posteriorly. The middle section was excised in total ensuring mucosa and turbinate bone remain intact and gently removed using endoscopic forceps. The specimens were immediately placed in ice cold $\left(4^{\circ} \mathrm{C}\right)$ culture medium and taken to the laboratory. The culture medium consisted primarily of Dulbecco Modified Eagle Medium/Ham Nutrient Mixture (Logan, UT, USA). Further details of cell culture are given in a previous publication ${ }^{(4)}$.

\section{Statistics}

Statistical tests were undertaken using Stata version 13.1 (StatCorp, TX, USA). Graphical presentations were performed using GraphPad Prism 5.0a (GraphPad Software, San Diego, CA, USA). The olfactory scores between groups were compared using 2-way analysis of variance, with turbinate biopsy and endoscopic sinus surgery as a source of variation. Analysis was performed first on the raw UPSIT scores as the primary endpoint. Scores were also adjusted for age and gender using percentile norms published by Doty et al. ${ }^{(20)}$.

The impact of secondary endpoints (Lund-Kennedy endoscopic outcome, SNOT-22, VAS, NOSE score) were sought by 2-way ANOVA. Correlation between pyschophysical olfactory score assessed by UPSIT and subjective patient's perception assessed by visual analogue scale on sense of smell was analysed using Spearman regression.

Effects of cofactors such as smoking, sex and polyposis on baseline UPSIT scores were analysed using U Mann Whitney test. 
Table 1. Population demographic and comparison with Sup' turbinate biopsy.

\begin{tabular}{|c|c|c|c|}
\hline & Patients Data & Biopsy & No biopsy \\
\hline \multirow[t]{3}{*}{ Demographic data } & Total, No & 65 & 66 \\
\hline & Age, mean $( \pm S D)$, yr & $47.2( \pm 14.5)$ & $46.7( \pm 15.9)$ \\
\hline & Sex, No (\%) & $\begin{array}{l}24 \mathrm{M}(36.9 \%) \\
41 \mathrm{~F}(63.1 \%)\end{array}$ & $\begin{array}{l}30 \mathrm{M}(45.4 \%) \\
36 \mathrm{~F}(54.6 \%)\end{array}$ \\
\hline \multirow[t]{17}{*}{ Surgical indication } & CRS with Polyps, No (\%) & $23(35.4 \%)$ & $32(48.5 \%)$ \\
\hline & CRSsP, No (\%) & $21(32.3 \%)$ & $21(31.8 \%)$ \\
\hline & Antro-choanal Polyp, No (\%) & $6(9.2 \%)$ & $3(4.5 \%)$ \\
\hline & Inverted papilloma & $5(7.7 \%)$ & $2(3.0 \%)$ \\
\hline & Foreign body, No (\%) & $3(4.6 \%)$ & $1(1.5 \%)$ \\
\hline & Fungocele / Mucocele, No (\%) & $2(3.1 \%)$ & $1(1.5 \%)$ \\
\hline & Spheno-palatine ligation, No (\%) & $0(0.0 \%)$ & $1(1.5 \%)$ \\
\hline & Amyloidosis, No (\%) & $1(1.5 \%)$ & $0(0.0 \%)$ \\
\hline & Sarcoidosis, No (\%) & $0(0.0 \%)$ & $1(1.5 \%)$ \\
\hline & $\begin{array}{l}\text { Ethmoidal adenocarcinoma, No } \\
(\%)\end{array}$ & $1(1.5 \%)$ & $0(0.0 \%)$ \\
\hline & Maxillary hypoplasia, No (\%) & $0(0.0 \%)$ & $1(1.5 \%)$ \\
\hline & Oro-antral fistula, No (\%) & $0(0.0 \%)$ & $1(1.5 \%)$ \\
\hline & Infected Concha bullosa, No (\%) & $1(1.5 \%)$ & $0(0.0 \%)$ \\
\hline & CSF leak, No (\%) & $0(0.0 \%)$ & $1(1.5 \%)$ \\
\hline & Sphenoid fungus ball & $0(0.0 \%)$ & $1(1.5 \%)$ \\
\hline & Pott's puffy tumour & $1(1.5 \%)$ & $0(0.0 \%)$ \\
\hline & Frontal sinus stenosis & $1(1.5 \%)$ & $0(0.0 \%)$ \\
\hline \multirow[t]{2}{*}{ Comorbidities } & Revision surgery, No (\%) & $8(12.3 \%)$ & $8(12.1 \%)$ \\
\hline & Smoking, No (\%) & $16(24.6 \%)$ & $10(15.2 \%)$ \\
\hline CT-scan & Lund-Mackay score, mean ( \pm SD) & $9.7( \pm 6.7)$ & $11.8( \pm 6.9)$ \\
\hline
\end{tabular}

Patient demographics.

Linear regression analysis was used to calculate the correlation between age and the baseline UPSIT score. Interaction of cofactors on the olfactory results of sinus surgery was evaluated using 2-way ANOVA.

\section{Sample size calculation}

All patients were consented for the procedure and refusal for the biopsy allowed for potential entry into the control arm. Sample size calculation of 41 patients in each arm was deemed satisfactory to provide a significant result although a larger sample was deemed ideal to account for drop out. Using data from the pilot series of patients, 7 pre-op and 4 post-op UPSIT scores, average change in mean UPSIT is 0.33 , sd 3.2, to detect a difference in score change of 0.33 in control group and 3 in olfactory biopsy group, power $80 \%, P=0.05$, requires 31 patients in pre-operative and post-operative groups. Allowing for loss to follow-up of 10 patients, a sample size of 41 patients was required for each group.

\section{Results}

Our population, summarized in Table 1, was representative of the population undergoing sinus surgery in a tertiary referral hospital. Out of the 136 patients for whom inclusion in our study was offered, 131 completed the UPSIT test and were randomized for superior turbinate biopsy, 2 could not complete the UPSIT test due to language barrier, 1 did not have time to complete the UPSIT test, 1 patient declined to participate and 1 planned intervention was cancelled. Out of the 131 subjects 
Table 2. Outcome data.

\begin{tabular}{|c|c|c|c|c|}
\hline & Surgical Response & pre-operative & post-operative & $\mathbf{p}$ \\
\hline \multirow[t]{3}{*}{ UPSIT (value) } & Whole population, mean $( \pm S D)$ & $25.9( \pm 9.6)$ & $26.7( \pm 9.7)$ & $0.3 \mathrm{~ns}$ \\
\hline & Superior turbinate biopsy & $26.6( \pm 9.3)$ & $27.9( \pm 8.8)$ & $0.2 \mathrm{~ns}$ \\
\hline & No biospy & $25.2( \pm 9.9)$ & $25.1( \pm 10.7)$ & $0.9 \mathrm{~ns}$ \\
\hline \multirow[t]{3}{*}{ UPSIT (percentile) } & Whole population, mean $( \pm S D)$ & $17.5( \pm 23.3)$ & $19.4( \pm 22.3)$ & $0.3 \mathrm{~ns}$ \\
\hline & Superior turbinate biopsy & $17.0( \pm 19.4)$ & $20.1( \pm 21.6)$ & $0.5 \mathrm{~ns}$ \\
\hline & No biospy & $18.0( \pm 26.9)$ & $18.8( \pm 22.7)$ & $1.0 \mathrm{~ns}$ \\
\hline \multirow[t]{3}{*}{ SNOT-22 score } & Whole population, mean ( \pm SD) & $51.9( \pm 25.4)$ & $29.1( \pm 24.2)$ & $<0.0001^{* * *}$ \\
\hline & Superior turbinate biopsy & $54.8( \pm 24.5)$ & $32.7( \pm 26.9)$ & $<0.0001^{* * *}$ \\
\hline & No biospy & $48.9( \pm 26.2)$ & $25.1( \pm 20.3)$ & $<0.0001^{* * *}$ \\
\hline \multirow[t]{3}{*}{ Lund-Kennedy score } & Whole population, mean $( \pm$ SD) & $4.4( \pm 2.7)$ & $2.1( \pm 1.6)$ & $<0.0001 * * *$ \\
\hline & Superior turbinate biopsy & $4.2( \pm 2.7)$ & $2.5( \pm 1.8)$ & $0.0002 * * *$ \\
\hline & No biospy & $4.6( \pm 2.7)$ & $1.5( \pm 1.3)$ & $<0.0001 * * *$ \\
\hline \multirow[t]{3}{*}{$\begin{array}{l}\text { VAS score on overall nose } \\
\text { symptom }\end{array}$} & Whole population, mean $( \pm$ SD) & $6.5( \pm 2.8)$ & $3.3( \pm 2.7)$ & $<0.0001 * * *$ \\
\hline & Superior turbinate biopsy & $6.8( \pm 2.6)$ & $3.4( \pm 3.1)$ & $0.0008 * * *$ \\
\hline & No biospy & $6.2( \pm 3.0)$ & $3.3( \pm 2.2)$ & $0.0005 * * *$ \\
\hline \multirow[t]{3}{*}{$\begin{array}{l}\text { VAS score on sense of } \\
\text { smell }\end{array}$} & Whole population, mean ( \pm SD) & $5.9( \pm 3.5)$ & $3.6( \pm 3.3)$ & $<0.0001^{* * *}$ \\
\hline & Superior turbinate biopsy & $5.6( \pm 3.4)$ & $3.6( \pm 3.3)$ & $0.0049 * *$ \\
\hline & No biospy & $6.3( \pm 3.7)$ & $3.7( \pm 3.4)$ & $0.0066 * *$ \\
\hline \multirow[t]{3}{*}{ NOSE score } & Whole population, mean ( \pm SD) & $12.9( \pm 5.9)$ & $5.6( \pm 4.9)$ & $<0.0001^{* * *}$ \\
\hline & Superior turbinate biopsy & $13.8( \pm 5.9)$ & $5.8( \pm 5.5)$ & $0.0004 * * *$ \\
\hline & No biospy & $12.3( \pm 5.9)$ & $5.3( \pm 4.3)$ & $0.0018 * *$ \\
\hline
\end{tabular}

UPSIT measurement, SNOT-22 score, endoscopic evaluation, visual analogue scale evaluation and NOSE scores were comparable in the superior turbinate biopsy group and in the control group and measurements outlined.

included in our study, 65 underwent a superior turbinate biopsy and 66 were randomized for the control group. Demographic data, severity of disease, co morbidities, incidence of smoking and surgical indications were comparable in the two groups as shown in Table 1.

A significant difference was not demonstrated between the two groups for baseline UPSIT scores as shown in Table 2. Biopsy of the superior turbinate did not affect the UPSIT result $(p=0.1468)$ and had no interaction with sinus surgery $(p=0.6376)$ as shown in Figure 1. There was no difference between biopsy and control groups for UPSIT scores. Adjustment for age and sex using UPSIT percentiles did not affect these findings. The evaluation of the sense of smell on a visual analogue scale subjectively improved in both groups after sinus surgery as shown in Table 2 . There was no negative impact of the olfactory mucosal biopsy.
A significant difference between the two groups in terms of secondary endpoint measures (Lund Kennedy endoscopic outcome, SNOT-22, VAS, NOSE score) was not demonstrated. There were no differences between the groups with respect to quality of life, patient's reported olfactory measure or endoscopic evaluation. However, there was also no correlation between the subjective sense of smell reported on a visual analogue scale and the objective evaluation by UPSIT. Superior turbinate biopsy did not affect the subjective sense of smell $(p=0.5920)$ and had no interaction with sinus surgery on 2 way ANOVA $(p=0.6835)$ as shown in Figure 2.

Superior turbinate biopsy had no affect on surgeon reported nasal mucosa outcomes, nasal blockage, overall nasal well being or quality of life. Equally it did not increase primary or secondary haemorrhage rate or intra-operative cerebrospinal leakage rate 


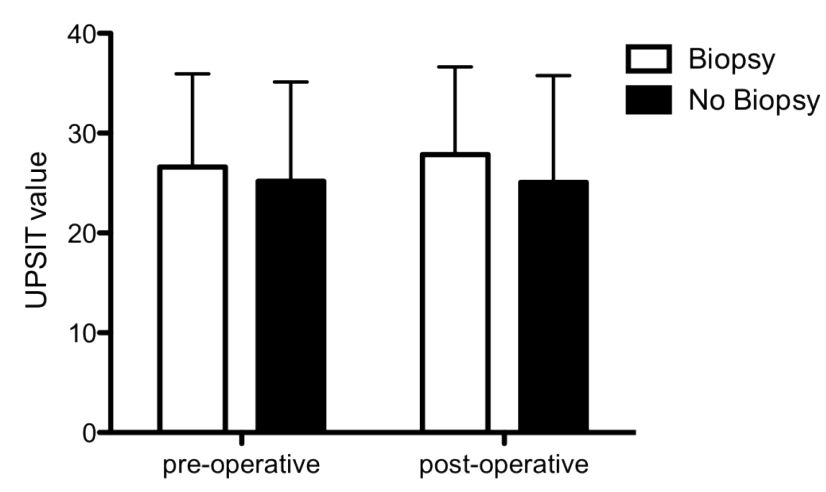

Figure 1. Effect of the superior turbinate biopsy on UPSIT value. Analysis by 2-way ANOVA. Biopsy has no interaction with FESS $(p=0.6)$. Biopsy does not affect the UPSIT value $(p=0.1)$. FESS does not affect the UPSIT value $(p=7)$.

when compared to the control group. The incidence of CSF leak was $0 \%$ in both groups.

\section{Discussion}

Patient safety, morbidity and nasal function, including olfactory outcomes, are not affected by unilateral resection of the middle section of the superior turbinate when compared to the outcomes of standard endoscopic sinus surgery without biopsy. Importantly we have uniquely demonstrated that the patient's quality of life and nasal patency remain unaffected following olfactory mucosa harvesting. This analysis has not been performed before and will aid in the process of informed consent.

One of our main concerns following the resection of the middle section of the superior turbinate was a potential reduction in the patient's sense of smell owing to the inevitable loss of olfactory mucosa. A perceived or measured olfactory loss was not demonstrated in this study and hence we can assume the olfactory loss was not significant enough for the patient to notice, which is in keeping with previous smaller studies ${ }^{(5,26)}$. Interestingly the perceived olfactory ability from healthy individuals and rhinological patients is generally poor. A recent study demonstrated even amongst healthy well functioning cognitive middle aged adults that $79 \%$ of this sample, with objectively assessed olfactory dysfunction, reported normal olfactory function ${ }^{(27)}$, A similar but less dramatic finding was also found in patients with rhinological pathology whereby $33 \%$ of patients who felt they had a normal sense of smell actually were hyposmic ${ }^{(28)}$.

The patient's subjective sense of smell actually improved significantly in both the biopsy and non biopsy groups which is to be expected given the majority of our patients suffered with CRS and presented with moderate to severe hyposmia. Current

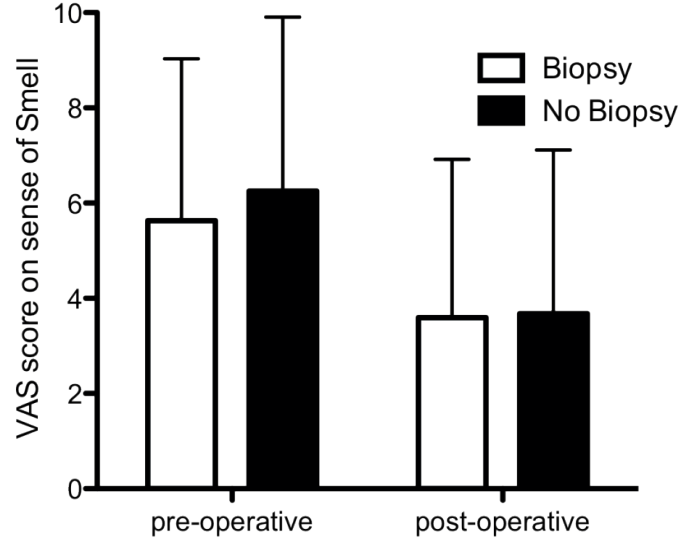

Figure 2. Effect of the superior turbinate biopsy on Visual Analogue Scale on sense of Smell. Analysis by 2-way ANOVA. Biopsy has no interaction with FESS $(p=0.7)$. Biopsy does not affect the VAS on sense of Smell $(p=0.6)$. FESS affects the VAS on sense of Smell significantly $(p<0.0001)$ *** (improvement).

evidence supports the efficacy of FESS surgery in improving the sense of smell in CRS patients albeit not back to normality whereby the majority still remain hyposmic post operatively ${ }^{(29)}$. Although the UPSIT score or psychophysical sense of smell improved in both arms it was not significant and a correlation between the patient's UPSIT outcomes and respective smell VAS scores was not found. As to why the VAS and UPSIT scores did not correlate is unclear but it may reflect the heterogeneous nature of the study group. Therefore, it must be stressed that one of the limitations of this study is a significant selection bias whereby the majority of the patients have suffered with CRS and would therefore expect to demonstrate an improvement in olfaction following FESS. This may have over-shadowed any potential olfactory loss as a result of olfactory biopsy although having a control arm has provided a well matched comparison.

Over $75 \%$ of our recruited patients were diagnosed with chronic rhinosinusits with or without nasal polyposis, in accordance with the European Position Paper on Rhinosinusitis and Nasal Polyps (EPOS) guidelines ${ }^{(19)}$. These patients present with a more predictable improved olfactory outcome, particularly in the CRSWNP subgroup ${ }^{(30)}$, when compared to the non-CRS group. Olfactory cleft opacification on CT scanning as well as Lund and Mackay scores negatively correlate with improved olfactory outcomes which also help in predicting outcome and prognosis ${ }^{(31)}$. However, in the non-CRS cohort, the olfactory outcomes were less predictable owing to the nature of their pathology including neoplasia and CSF leak repair. Even so, the mixture of disease diagnoses were evenly distributed between each of the arms and equally the severity of the disease processes were also matched as depicted by the Lund Mackay scoring shown in Table 1.

An alternative hypothesis for the disconnect between the 


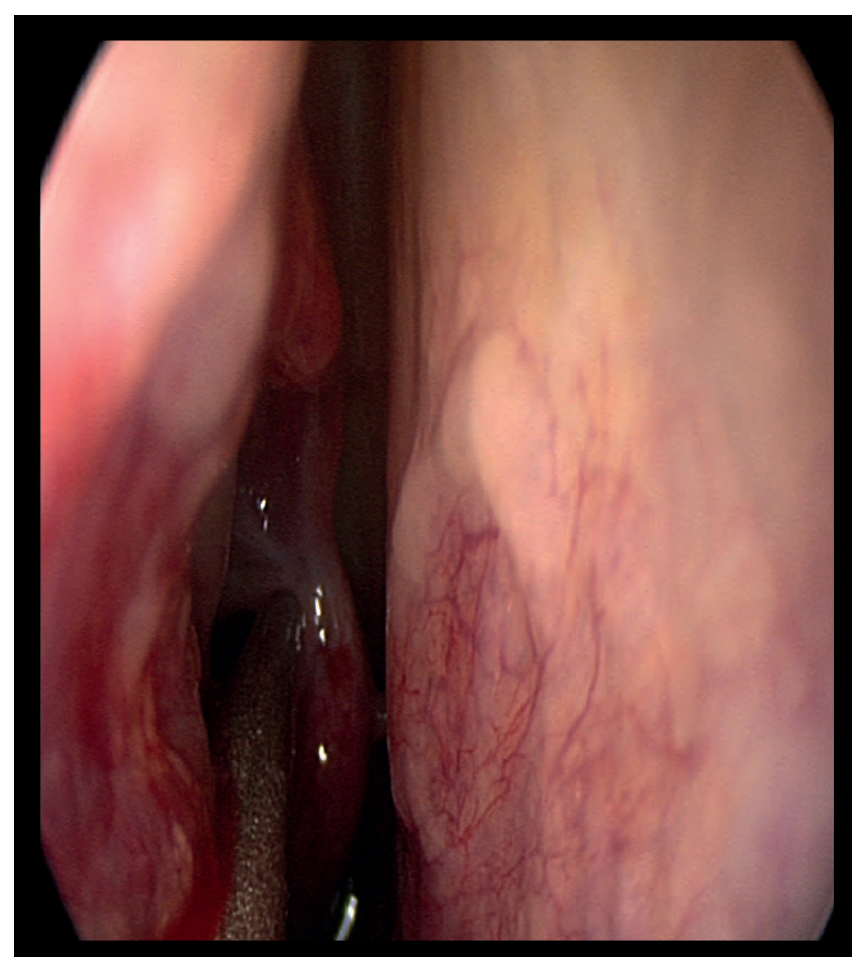

Flgure 3. Endoscopic photograph of superior turbinate 2 years following middle section resection.

two olfactory outcome measurements may have been due to the specificity of the UPSIT measurement itself which only measures olfactory identification as opposed to threshold and discrimination. In future studies we will incorporate an olfactory measurement which will measure all three olfactory outcomes. Equally it could be argued that by improving the patient's nasal symptoms and quality of life they would be more inclined to also feel their sense of smell has improved. Although we have demonstrated the beneficial outcomes following harvesting human olfactory nasal mucosa during Functional Endoscopic Sinus Surgery (FESS) are statistically the same when compared to the outcomes of standard FESS without biopsy, the question still remains, as to what would happen to olfaction following olfactory biopsy in normal and non-diseased individuals.

In addition it could be argued that the improved sense of smell which was demonstrated in the biopsy group may have occurred as a result of subsequent regeneration of the biopsied area, with a repopulation of olfactory epithelium. However, this remains doubtful owing to the lack of regeneration of the biopsied middle section of the superior turbinate as seen in Figure 3. In this figure you can see the deficient middle section with a small adhesion as seen 2 years post biopsy. Although the nasal mucosa has regenerated over the defect, the surface area remains deficient owing to the lack of underlying turbinate architecture.
The biopsy size taken in this study equates to less than $5 \%$ of the total olfactory mucosa which does not appear to incur a noticeable olfactory loss to the patient. Interestingly the biopsy size taken in this study is in keeping with other biopsy sizes harvested in previous studies where loss of sense of smell was not demonstrated ${ }^{(5)}$. We estimated our biopsy size on histological analysis; whereby the average surface area of the olfactory mucosa biopsy measured 3-5mm squared, which included the whole of the middle section of the superior turbinate ${ }^{(4,5)}$.The average surface area of the human olfactory mucosa is between 10 and $20 \mathrm{~mm}$ squared in each cavity ${ }^{(32)}$. According to our results, a $5 \%$ loss of olfactory tissue does not incur a significant measured olfactory deficit however, the question remains as to how much could be harvested before the patient starts to notice deterioration.

All specimens underwent both histological and cell culture analysis and were all deemed suitable for culture. All our biopsied samples cultured OECs however there was a significant variation in yield rate which was dependent on mucosal disease and age. OEC yield rate was reduced with increased mucosal disease and an increase in patient age of the sample. The key was to ensure the mucosa remained attached to the underlying turbinate bony architecture which was the case in all specimens. The mean proportion of OECs per $1 \mathrm{~mm}$ of culture dish was $7.94 \%$, with the majority of the biopsies (48\%) yielding less than $5 \%$. Only $23 \%$ of these samples yielded OEC proportions of more than $50 \%{ }^{(4)}$. It was generally concluded that OEC yield rates were higher when biopsied from younger and non-diseased patients. Histological analysis was also performed on 34 of the biopsied samples and 32 stained positive to OEC olfactory bundles. This olfactory mucosa positivity proved to be higher when compared to other similar studies ${ }^{(26,33)}$.

Resection of the middle section of the superior turbinate did not result in a single peri-operative CSF leak as demonstrated by our results and the surgical procedure for this technique has already been described in the literature ${ }^{(4)}$. However prior to this study and during the development of this technique, resection of a different region (the superior section of the superior turbinate) was trialed and did result in a single CSF leak, which was successfully repaired and consequently this initial technique was abandoned. We therefore advise against harvesting the superior section of the superior turbinate, owing to its close proximity to the anterior skull base.

One of the limitations of this study lies in the subjective nature of measuring olfaction. The evaluation of patient's sense of smell was subjectively measured using both the UPSIT and VAS scoring methods and as a result were both open to potential patient bias. The UPSIT test was chosen in this study owing to its 
universal track record and the validation of the British version ${ }^{(34)}$ It is the most validated chemosensory and psychophysical technique used internationally and has the largest series of robust objective correlations as well as evaluating the probability of malingering in our patient group. As a forced response test from 4 potential answers, there is a 1 in 4 chance of getting a correct answer and hence a score below 8 increases the probability of malingering as well as testing cognition at the same time ${ }^{(20)}$.

Sniffin sticks are the alternative psychophysical olfactory measurement which have been validated in the UK and can be additionally used to determine olfactory thresholds and discrimination acuity which may prove to be more sensitive for future studies ${ }^{(35)}$. There is also an argument for testing olfaction unilaterally as opposed to bilaterally, however, we felt that if a difference were to be seen it would be picked up in either case and testing individual nostrils would add to the duration and compliance of the test ${ }^{(36)}$.

The feeling of blockage or other associated nasal blockage outcomes as measured by the NOSE questionnaire and the overall VAS score reassures us that this procedure does not affect the nasal airway or patient's sleep or exercise. A potential consequence of this procedure may have been excessive crusting or scarring but this was not the case whereby the surgeon reported outcomes depicted good healing in both arms. Importantly the incidence of crusting or adhesions was not increased in the biopsy arm. The SNOT 22 quality of life score equally improved in both arms and did not show a difference between the two which reassures us that the superior turbinate biopsy does not affect the quality of life of our patients.
This olfactory harvesting technique is safe and reproducible and does not incur an olfactory or nasal function deficit and can be rolled out and safely used in other olfactory therapeutic and diagnostic studies including the early detection of neurodegenerative diseases ${ }^{(37,38)}$.

\section{Conclusion}

Our results provide level 2 a evidence which demonstrates unaffected patient safety, without increased morbidity, following harvest of human olfactory nasal mucosa. We have uniquely shown that endoscopic biopsy of olfactory mucosa does not affect nasal function or the sense of smell compared to standard FESS without biopsy. This comparison of olfactory harvesting surgery with a control group from a large cohort of patients undergoing endoscopic sinus surgery allows us to inform and consent patients appropriately.

\section{Acknowledgements}

We acknowledge research funding from the European Research Council. This work was conducted in part at the UCL Institute of Neurology, which receives funding from the National Institute for Health Research Biomedical Research Centre.

\section{Authorship contribution}

Study design, patients' care and surgical procedures were performed by PA. PA, ALP and DC collected the data and were involved in literature search. Data interpretation was performed by PA and ALP. Writing was performed by PA, DC, ALP and VL.

\section{Conflict of interest}

No conflict of interest to disclose.

\section{References}

1. Kafo S, Choi D. Clinical studies in spinal cord injury: moving towards successful trials. $\mathrm{Br}$ Neuosurg 22, 3-12. 2008.

2. Mackay-Sim A, St John JA. Olfactory ensheathing cells from the nose: clinical application in human spinal cord injuries. Exp Neurol 229, 174-180. 2011.

3. Feron F, Cochrane J, Perry C, et al. Autologous olfactory ensheathing cell transplantation in human spinal cord injury. Brain 2005;128:2951-60.

4. Kachramanoglou C, Law S, Andrews P, Li D, Choi D. Culture of olfactory ensheathing cells for central nerve repair: the limitations and potential of endoscopic olfactory mucosal biopsy. Neurosurgery 7, 170-179. 2013

5. Lanza DC, Deems DA, Doty RL, et al. The effects of human olfactory biopsy on olfaction: A preliminary report. Laryngoscope
104, 837-840. 1994.

6. Feron F, Perry C, Hirning M, McGrath J, Mackay-Sim A. Altered adhesion, proliferation and death in neural cultures from adults with schizophrenia. Schizophr Res 40[211], 218. 1999.

7. Li Y, Field PM, Raisman G. Regeneration of adult rat corticospinal axons induced by transplanted olfactory ensheathing cells. J Neurosci 18(24), 10514-10524. 1998.

8. Li Y, Field PM, Raisman G. Olfactory Ensheathing Cells and Olfactory Nerve Fibroblasts Maintain Continuous Open Channels for Regrowth of Olfactory Nerve Fibres. Glia 52, 245-251. 2005.

9. Li Y, Sauvre Y, Li D, Lund RD, Raisman G. Transplanted olfactory ensheathing cells promote regeneration of cut adult rat optic nerve axons. J Neurosci. 23(21), 7783-7788. 2003.

10. Bianco Jl, Perry C, Harkin DG, Kay-Sim A,
Feron F. Neurotrophin 3 promotes purification and proliferation of olfactory ensheathing cells from human nose. Glia 45(2), 111 123. 2004.

11. Mackay-Sim A, Feron F, Cochrane J, Bassingthwaighte L, Bayliss C, ea. Autologous olfactory ensheathing cell transplantation in human paraplegia: A 3-year clinical trial. Brain 131, 2376-2386. 2008.

12. Doucette R. Glial influences on axonal growth in the primary olfactory system. Glia 3, 433-449. 1990.

13. Barnet SC, Hutchins AM, Noble $M$. Purification of olfactory nerve ensheathing cells from the olfactory bulb. Dev Biol 155, 337-350. 1993.

14. Doucette R. The glial cells in the nerve fibre layer of the rat olfactory bulb. Anat. Rec. 210, 385-391. 1984.

15. Raisman G. Specialized neuroglial arrange- 
ment may explain the capacity of vomeronasal axons to reinnervate central neurons. Neurosci 1985;14(1):237-54.

16. Lindsay SL, Riddell JS, Barnett SC. Olfactory mucosa for transplant-mediated repair: a complex tissue for a complex injury? Glia, 125-134. 2010.

17. Franklin RJ, Gilson JM, Franceschini $\mid A$, Barnett SC. Schwann cell-like myelination following transplantation of an olfactory bulb ensheathing cell line into areas of demyelination in the adult CNS. Glia 17 217-224. 1996.

18. Kachramanoglou C, Li D, Andrews , et al. Novel strategies in brachial plexus repair after traumatic avulsion. Br J Neurosurg 25, 16-27. 2011.

19. Fokkens WJ, Lund VJ, Mullol J, Bachert C, et al. EPOS 2012: European position paper on rhinosinusitis and nasal polyps 2012. A summary for otorhinolaryngologists. Rhinology. 50[1], 1-12. 2012.

20. Doty $R L$, Shaman $P$, Kimmelman $C P$ Dann MS. University of Pensylvania Smell Identification Test: a rapid quantitative olfactory function test for the clinic. Laryngoscope 94, 176-178. 1984.

21. Lund VJ, Kennedy DW. Quantification for staging sinusitis. In: Kennedy DW, editor. International Conference on Sinus Disease: Terminology, Staging, Therapy. Ann Otol Rhinol Laryngol 104(suppl 167), 17-21. 1995.

22. Hopkins C, Gillet S, Slack R, Lund V, Browne JP. Psychometric validity of the 22-item Sinonasal Outcome Test. Clin Otolaryngol. 34, 447-453. 2009

23. Lund VJ, Mackay IS. Staging in chronic rhinosinusitis. Rhinology 1993;31:183-4.

24. Poirrier AL, Ahluwalia S, Goodson A, Ellis M, Bentley M, Andrews P. Is the Sino-Nasal Outcome Test-22 a suitable evaluation for septorhinoplasty? Laryngoscope. 123[1],
76-81. 2013

25. Stewart MG, Witsell DL, Smith TL, Weaver DL, Yueh B, Hannley MT. Development and validation of the Nasal Obstruction Symptom Evaluation (NOSE) scale. Otolaryngol Head Neck Surg 130[2], 157163. 2004.

26. Winstead W, Marshall CT, Lu CL, Klueber KM, Roisen FJ. Endoscopic biopsy of human olfactory epithelium as a source of progenitor cells. Am J Rhinol 2005;19(1):83-90.

27. Wehling $E$, Lundervold AJ, Espeset $T$, Reinvang I, Bramerson A, Nordin S. Even cognitively well-functioning adults are unaware of their olfactory dysfunction: Implications for ENT clinicians and researchers. Rhinology. 53[1], 89-94. 2015.

28. Philpott CM, Rimal D, Tassone P, Prinsley PR, Premachandra DJ. A study of olfactory testing in patients with rhinological pathology in the ENT clinic. Rhinology. 46[1], 34-39. 2008.

29. Briner HR, Jones $N$, Simmen D. Olfaction after endoscopic sinus surgery: long-term results. Rhinology. 50[2], 178-184. 2012.

30. Lind $H$, Joergensen $G$, Lange $B$, Swendstrup $F$, Kjeldsen AD. Efficacy of ESS in chronic rhinosinusitis with and without nasal polyposis: a Danish cohort study. Eur Arch Otorhinolaryngol 273[4], 911-919. 2016.

31. Vandenhende-Szymanski C, Hochet B Chevalier D, Mortuaire G. Olfactory cleft opacity and CT score are predictive factors of smell recovery after surgery in nasal polyposis. Rhinology. 53[1], 29-34. 2015.

32. Gray H. Grays Anatomy (The Classic Collectors Edition). 1977.

33. Say P, Leopolod DCG, Smith L, Greiner T. Resection of the inferior superior turbinate: does it affect olfactory ability or contain olfactory neuronal tissue? Am J Rhinol 2004;18(3):157-60.
34. Muirhead N, Benjamin E, Saleh H. Is The University Of Pennsylvania Smell Identification Test (UPSIT) Valid for the UK Population? Otolaryngologist 6[2], 99-103. 2013.

35. Neumann C, Ksioulos K, Merkonidis C Salam M, Clark A, Philpot C, et al. Validation study of the "Sniffin' Sticks" olfactory test in a British population: a preliminary communication. Clin Otolaryngol 37[1], 23-27. 2012.

36. Welge-Lussen A, Gudziol V, Wolfensberger M, Hummel T. Olfactory testing in clinical settings - is there additional benefit from unilateral testing? Rhinology 2010:48(2):156-9.

37. Randhawa PS, Watson N, Lechner M, Ritchie $L$, Choudhury N, Andrews P. The outcome of septorhinoplasty surgery on olfactory function. Clin Otolaryngol. 41[1], 15-20. 2016.

38. Schneider SA, Boettenger M, Alexoudi A, Zorenkov D, Deuschl G, Wedwl T. Can we use peripheral tissue biopsies to diagnose Parkinson's disease? A review of the literature. Eur J Neurol. 23, 2, 247-261. 2016.

Mr Peter Andrews Bsc. FRCS.

11 Elliswick Road

Harpenden

Hertfordshire Al54TP

United Kingdom

Tel : +44-794-054 9414

E-mail: peterandrews2@gmail.com 